LA-UR- $94-4163$

TITLE: RICH CLUSTER SIMULATION: DYNAMICS AND MASS ESTIMATES

AUTHOR(S): Benjamin Bromley, T-6

Michael Warren, T-3

Wojciech Zurek, T-6

P. J. Quinn, Mount Stromlo Observatory

SUBmitted To: $\quad$ 5TH Annual Astrophysics Conference, College Park, MD, October 10-12

By acceptance of this article, the publisher recognizes that the U.S. Government retains a nonexclusive, royalty-iree license to publish or reproduce the published form of this contribution, or to allow others to do so, for U.S. Government purposes.

The Los Alamos National Laboratory requests that the publisher identify this article as work performed under the auspices of the U.S. Department of Energy.

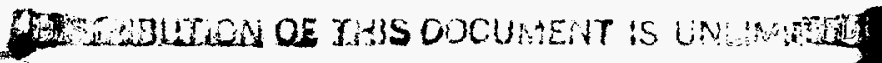
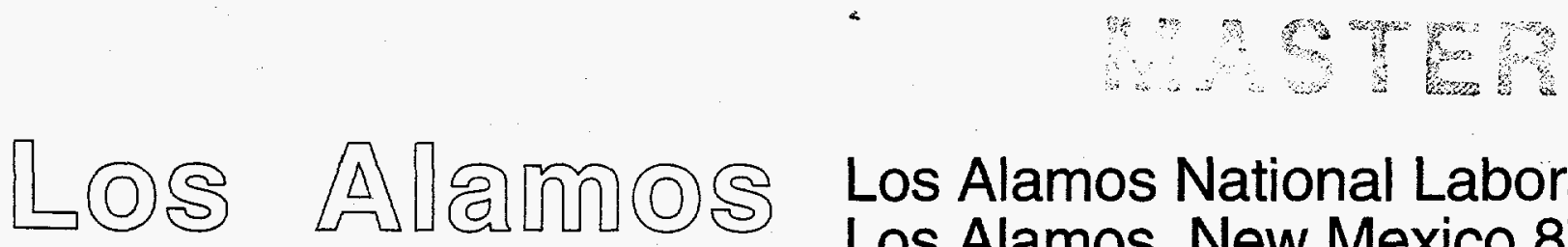

Los Alamos National Laboratory
Los Alamos, New Mexico 87545 


\section{DISCLAIMER}

This report was prepared as an account of work sponsored by an agency of the United States Government. Neither the United States Government nor any agency thereof, nor any of their employees, make any warranty, express or implied, or assumes any legal liability or responsibility for the accuracy, completeness, or usefulness of any information, apparatus, product, or process disclosed, or represents that its use would not infringe privately owned rights. Reference herein to any specific commercial product, process, or service by trade name, trademark, manufacturer, or otherwise does not necessarily constitute or imply its endorsement, recommendation, or favoring by the United States Government or any agency thereof. The views and opinions of authors expressed herein do not necessarily state or reflect those of the United States Government or any agency thereof. 


\section{DISCLAIMER}

Portions of this document may be illegible in electronic image products. Images are produced from the best available original document. 


\title{
RICH CLUSTER SIMULATION: DYNAMICS AND MASS ESTIMATES
}

\author{
B.C. Bromley, M.S. Warren, W.H. Zurek, \\ Theoretical Astrophysics, Los Alamos National Laboratory \\ P.J. Quinn \\ Mount Stromlo Observatory
}

\begin{abstract}
We present an analysis of a simulated rich cluster dominated by cold dark matter. Here, we focus on the dynamics of collapsed dark matter halos which may be associated with galaxies. We use projected positions and velocities of both dark matter particles and of halos to assess several conventional measures of cluster mass. The results indicate that the measures give reasonable results for dark matter particles but cause overestimates when applied to halos. In searching for the source of the discrepancies, we find that tidal disruption and dynamical friction do not induce a significant bias in the projected velocity disperion of halos; however, an antibias in the spatial distribution of halos relative to that of the total mass appears to be most problematic for cluster mass estimates.
\end{abstract}

\section{INTRODUCTION}

Galaxy clusters have played a key role in the effort to determine the nature of matter in the Universe and the value of the cosmological density parameter $\Omega$. The dynamical mass of clusters has particularly important cosmological implications, as first recognized when Zwicky (1933) deduced presence of dark matter in the Coma cluster. Several estimators designed to yield cluster mass from projected galaxy velocities have been introduced by Bacall \& Tremaine (1981) and Heisler, Tremaine \& Bahcall (1985). These authors assessed such measures in numerical $N$-body simulations with 10 or less point-like galaxies. Subsequently, West \& Richstone (1988) performed tests with simulations of $N=10^{4}$ particles, although galaxies were still approximated as point particles.

Ideally, mass estimates should be tested with simulations that include the large-scale gas dynamics of the baryons as well as high mass resolution to track the interaction of extended galactic halos with the rest of the cluster. However, work on gas dynamics within clusters (e.g., Evrard 1990) indicates that cooling processes may be neglected in the large-scale intracluster gas-the baryonic component may be effectively dissipationless. Thus as an approximation, dissipative effects may be neglected when one proceeds to higher and higher mass resolution.

Here we present the results of a million-particle high-resolution simulation of a cluster with dissipationless cold dark matter. The cluster is dominated by a single massive core interspersed with smaller collapsed halos which may be associated with galaxies. We track the dynamical evolution of the halos, as well as that of their constituent dark matter particles. Our principal aim 
is to test conventional measures of cluster mass based on projected positions and velocities, as might be available to a distant observer. We first use the mass particles to test the assumptions, such as virialization, upon which these measures rest. Then, we focus upon the applicition of the mass measures to the halos. At issue are the effects of tidal disruption and dynamical friction, and the extent to which the halo phase-space distribution is biased with respect to that of the total mass.

\section{NUMERICAL METHOD}

The simulation of the cluster began with selection of a primordial overdense structure, the seed for a present-epoch cluster, from a palette of random realizations of the cosmic density field. Each realization was generated as a Gaussian field variate with a COBE-normalized CDM power spectrum (Holtzman 1989) in an $\Omega=1$ universe. The selected initial field was a 1 -in-500 event; it contained a $2.5-\sigma$ overdensity in the middle $1 / 9$ th of the simulation volume. Force calculation for this $1.1 \times 10^{6}$ particle system was performed using a tree code with spatial resolution of $5 \mathrm{kpc}$ (see Warren 1994). The final output, obtained after 3000 computational timesteps, had a radius of $15 \mathrm{Mpc}$ and a Hubble parameter of $50 \mathrm{~km} / \mathrm{s} / \mathrm{Mpc}$. The evolved cluster had a mass of approximately $5 \times 10^{14} \mathrm{M}_{\odot}$ within the Abell radius.

In the simulation, aggregates of dark matter particles were identified as galaxy halos with the algorithm described by Warren et al. (1992). The constituent particles of 40 halos were tagged and tracked from a redshift of $z=0.85$ to the present epoch $(z=0)$.

\section{CLUSTER MASS MEASURES}

We examine three conventional cluster-mass measures (Bahcall \& Tremaine 1981; Heisler et al. 1985; Binney \& Tremaine 1987) for a cluster of $N$ galaxies and a radius $R$ :

$$
\begin{gathered}
\text { Isothermal Mass : } 2 R \sigma_{1}^{2} / G ; \\
\text { Virial Mass : } 3 \pi N \sigma_{1}^{2} / 2 G R_{H} ; \\
\text { Projected Mass : } 32 \alpha<v_{\|}^{2} r_{\perp}>/ \pi G .
\end{gathered}
$$

In these expressions, $\sigma_{1}$ is the dispersion in the line-of-sight velocities $v_{\|} ; r_{\perp}$ and $R_{H}$ are radial distance from the cluster center and the mean harmonic radius, respectively, both measured in the sky plane of a distant observer. The factor of $\alpha$ in the projected-mass measure ranges from 1 for isotropic orbits to 2 for radial orbits.

These mass estimates are applied to the CDM particles in the simulation and are shown in Figure $1 \mathrm{a}$ as a function of plane-of-sky radius $r_{\perp}$. Evidently, the virial and projected mass estimates are in fair agreement with the true mass within the Abell radius. The isothermal mass estimate works well only within the core of the cluster.

When applied to the halo population, the mass estimates fare considerably worse (Fig. 1b). In general, the mass is overestimated by $40 \%$ to $250 \%$ for $1 \mathrm{Mpc}<R<3 \mathrm{Mpc}$. We now examine possible sources of the discrepancies. 


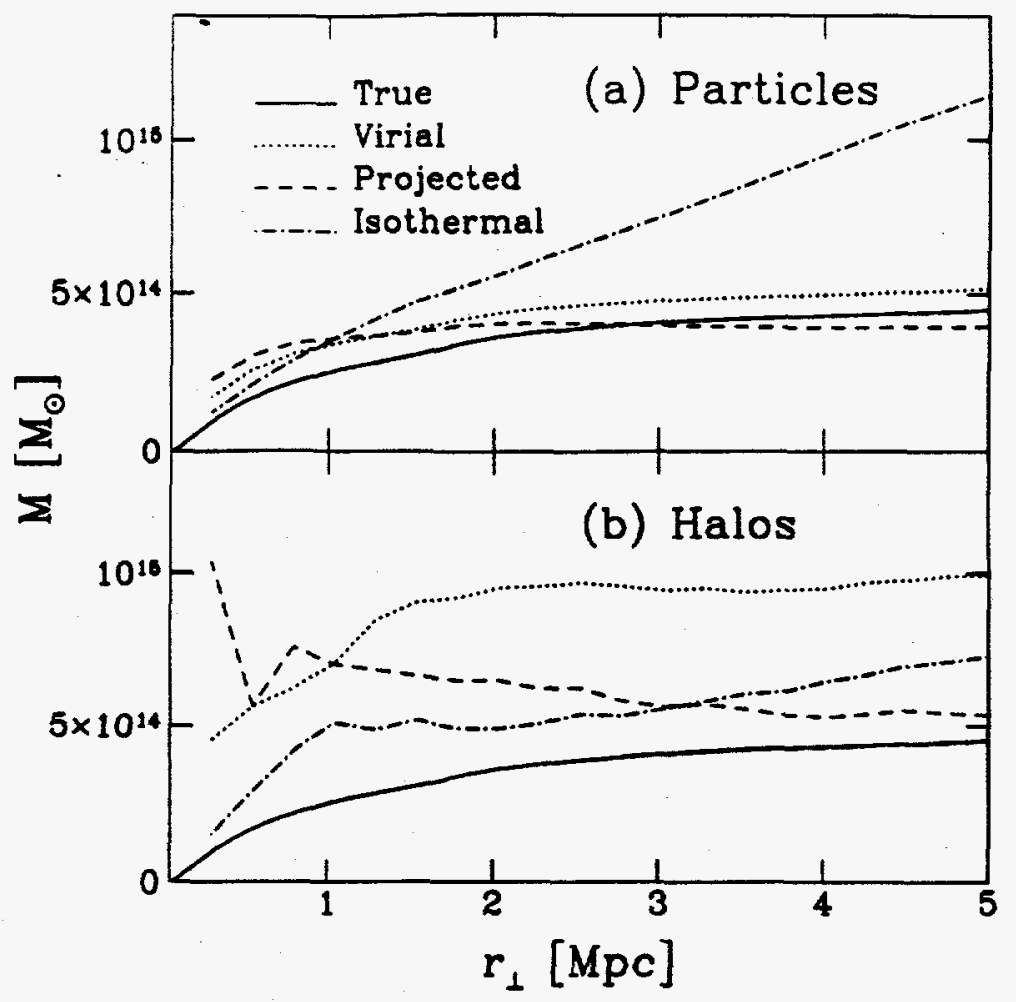

Fig. 1. Cluster mass measures from (a) total mass and (b) halos as a function of plane-of-sky radius $r_{\perp}$. The cluster was defined to include all objects within $7.5 \mathrm{Mpc}$ of the center of mass.

The mass and force resolution of the simulation are sufficiently high that we may expect to see the effects of tidal forces and dynamical friction on the halo trajectories. We found that tidal effects were not significant even in those orbits that passed very near the cluster core, as only $5 \%$ of the halos were disrupted.

The more subtle phenomenon of dynamical friction, which may result when halos have a large number of internal degrees of freedom, may produce a velocity bias in the halo trajectories. Such a bias has been reported in pairwise velocity statistics from cosmological simulations (Couchman \& Carlberg 1992). Here, we do not find evidence for a significant bias in the one-point halo velocity distribution. For example, the 1D halo velocity dispersion as a function of planeof-sky radius from the cluster core traces the dispersion profile for particles over a broad range of $r_{\perp}$. Thus, a velocity bias is not the source of error in the mass measures discussed above.

However a spatial bias is detected. Evidence appears in $R_{H}$, the mean harmonic radius in the sky plane. The profile of $R_{H}$ for halos lies significantly above that for particles, indicating that halos are more dilute in their contribution to the cluster potential than expected if they were a random subset of the total mass. An increase in $R_{H}$ at fixed velocity dispersion strengthens the magnitude of the inferred gravitational potential and drives the virial and projected mass measures upward from the true value. We conclude that spatial bias is the principal source of error in these measures. 


\section{CONCLUSION}

The small smoothing scale of $5 \mathrm{kpc}$ for the force calculation and the large number of partictes distinguish this experiment from previous numerical simulations of dissipationless cluster dynamics. We find that conventional mass measures based on projected velocities and postions are in reasonable agreement with the actual mass when simulation particles are used. However, these same measures generally overestimate the cluster mass when applied to halos.

This discrepancy is linked to the spatial antibias of the halo distribution. A halo velocity bias, such as might be expected if tidal disruption and dynamical friction were dominant processes, was not detected. These conclusions compare favorably, in qualitative terms, with analyses of $\mathrm{x}$-ray and optical data: Lubin \& Bahcall (1993) argue for an absence of a velocity bias, while Eyles et al. (1991) find that galaxies are antibiased in clusters and dynamical masses are overestimated. We must caution that in our data the spatial antibias may be artificially enhanced by the halo finding algorithm and by the dissipationless assumption.

In closing, we note that the spatial antibias of galaxies in clusters has ramifications for other cosmological measures. An example is the pairwise radial velocity dispersion, $\sigma_{12}$. Even if galaxies are exact tracers of the cosmic velocity field, $\sigma_{12}$ inherits a bias simply because the high-velocity galaxies in the dense clusters are under-represented compared with the slower objects in the field. This effect can be of considerable importance for cosmological information derive from $\sigma_{12}$, as discussed at this conference by W. Zurek and elsewhere (Zurek et al.1994).

\section{ACKNOWLEDGEMENTS}

We are grateful to the Aspen Center for Physics where part of this work was completed. This research was supported by the NASA HPCC program.

\section{REFERENCES}

Bahcall, J., \& Tremaine, S. 1981, ApJ, 244, 805

Binney, J., \& Tremaine, S. 1987, Galactic Dynamics (Princeton: Princeton University Press)

Couchman, H.M.P., \& Carlberg, R.G. 1992, ApJ, 389, 453

Evrard, A.E., 1990, ApJ, 363, 349

Eyles, C.J, Watt, M.P., Bertram, D., Church, M.J., Ponman, T.J., Skinner, G.K., \& Willmore, A.P., 1991, ApJ, 376, 23

Heisler, J., Tremaine, S., \& Bahcall, J. 1985, ApJ, 298, 8

Holtzman, J.A., 1989, ApJS, 71, 1

Lubin, L.M, \& Bahcall, N.A. 1993, ApJ, 415, L17

Warren, M.S., 1994, Ph.D. Thesis, UCSB

Warren, M.S., Quinn, P.J., Salmon, J.K., \& Zurek, W.H. 1992, ApJ, 399, 405

West, M.J., \& Richstone, D.O. 1988, ApJ, 335, 532

Zurek, W.H., Quinn, P.J., Salmon, J.K., Warren, W.S. 1994, ApJ, 431, 559

Zwicky, F. 1933, Helv. Phys. Acta, 6110 